published by my friend Dr. Treedie, in /cotic powers. In a short time their shin the eighth part of the Cyclopedia of Prac- becomes soft, and assumes a healthy aptical Iredicine, as well as in the equally pearance. The lobelia inflata is the plant valuable Dictionary of Practical Medicine, which gives the emetic quality to the wellby my friend Dr. Copland, Part I., this known Indian " black-drink." It also enters subject is most ably handled, and to these into many and various combinations in the works I beg to refer your readers.

It is my duty, though late, to acknowledge, that to Dr. Stokes, of Dublin, I am indebted for hints upon the appearance of the blood in typhus fever, which forced me, as conversant with chemistry, to undertake the chemical analysis of blood in typhus fever, and also in spasmodic cholera.

I remain, Sir,

Your most obedient humble servant, Sunderland, Féb. 16, 1833 .

W. Reid Clanny.

\section{THE LOBELIA INFLATA.}

\section{To the Editor of THE LANCET.}

Sir,-Permit me, through the medium of The Laxcer, to make a few observations on one of the most valuable plants which has yet been discovered, a plant calculated to relieve the sufferings of our fellow men to an equal, if not greater degree, than any hitherto known. I allude to the lobelia inflata. From long experience and patient investigation of the properties of this plant, both in England and America, I have no hesitation in saying that it merits the first place in the Pharmacopocia, and time will show that my opinion is not hastily formed. I need not repeat the high encomiums so recently passed on it by Dr. Elliotson, the most enterprising physician of the age; but as the plant is not generally known, and consequently but little used, it may not be uninteresting to many of your readers to be made acquainted with some particulars respecting its genus and natural bis. tory.

The lobelia inflata belongs to the 19th class syngenesia, order monogynia, of Linnæus. The genus contains a great variety of species, fifteen of which grow in the United States, all of them possessing medicinal powers, but the plant in question is the most active of its congenera. Its properties were totally unknown until I observed the cattle and horses searching after it in the spring. After being confined to dry food in the winter, many of them become " hide-bound," and affected with dandriff, and they will then eat the plant greedily, which brings on a powerful salivation, vulgarly called " the slavers." Sometimes, by eating too much, they are poisoned, and but for the use of turnips and cabbage leaves, which they devour with avidity, many would fall victims to its nar-
Indian practice of pbysic.

Its qualities are, -a most powerful and efficient emetic, narcotic, expectorant, antispasmodic, diuretic, and sialagogue. It contains an acrid principle, caoutchouc, and extractive. In its effects it acts very much like tobacco, but the action is more speedy and diffusible. Like most other medicinal plants, unless it be collected from its proper locality and elaboration of its sap, it is sure to fail in producing the de. sired effect. Its antispasmodic and seda. tive properties are so fugacious, that they are dissipated by hot infusion, and are likewise affected by light, whilst its narcotic qualities are retained. By exposing the tincture to the light, it is changed from a light green to a dark brown colour. When given in large doses, like other narcotics, it affects the brain, and produces a lasting. prostration of strength. Such effects, low. ever, do not take place when its antispas. modic and sedative properties are pre. served. Hence the disagreement among medical men as to the specific action of plants, - attributable to the slovenly man. ner in which the plants are collected, pre. served, and prepared. In proof of this I will instance the plant in question :- Seven or ten drops of the tincture, prepared by me, is acknowledged to be equal to a drachm and a half of that sold in some of the shops.

Its medical properties and uses.-Lobelia inflata has been given in asthma. I have found it (with few exceptions) a perfect specific in every case of pure asthma ; and, as Dr. Elliotson has justly observed, " all other remedies hitherto made use of in the above disease are as nothing compared with it." For twenty years $I$ have been in the habit of using it; and in chronic bronchitis, loss of voice, nervous cough, hooping-cough, catarrh, and other diseases of the bronchia and larynx, I have found it equally efficacious. 'There are other dis. eases of a more complicated nature, in which great good has resulted from its use, viz. convulsions, tetanus, hydrophobia, St. Vitus's dance, \&c. In conclusion I have only to observe, that though the plant has been known for many years to the Indian tribes of America, yet to me the Americans are indebted for the discovery of its medicinal properties. $*^{*} *$ (l'he remainder of this letter is an advertisement.-ED. L.) I am, \&c., Chartes Winteaw.

14, Finsbury Place South. 